\title{
Effect of silencing SLPI gene expression on differentiation of BeWo cells
}

\author{
Neelima P. Sidarthan ${ }^{1}$, Vijayakumar Govindaraj ${ }^{2}$, Mary Nirmala Sarkar ${ }^{2}$, A. J. Rao ${ }^{2, *}$ \\ ${ }^{1}$ School of Biomedical sciences, University of Queensland, St Lucia, Queensland 4072, Australia \\ ${ }^{2}$ Department of Biochemistry, Indian Institute of Science, Bangalore, India
}

Email address:

ajrao_2000@yahoo.com (A. J. Rao)

To cite this article:

Neelima P. Sidarthan, Vijayakumar Govindaraj, Mary Nirmala Sarkar, A. J. Rao. Effect of Silencing SLPI Gene Expression on Differentiation of BeWo Cells. Cell Biology. Vol. 1, No. 1, 2013, pp. 1-8. doi: 10.11648/j.cb.20130101.11

\begin{abstract}
In our previous studies which involved genomic profiling by DD-RTPCR and microarray during forskolin induced differentiation of uninucleated cytotrophoblasts into multinucleated syncytiotrophoblasts using BeWo chorionic carcinoma cells as a model, the expression of one of the transcripts namely, Secretory Leucocyte Protease Inhibitor (SLPI) was found to be very high (10-15 fold) in syncytiotrophoblasts compared to the expression in cytotrophoblasts. SLPI is a protein of $12 \mathrm{kDa}$ molecular weight and a variety of activities which include protease inhibition, anti inflammatory and anti microbial activity have been attributed to it. In view of this, an attempt was made to investigate the role of SLPI during differentiation of cytotrophoblasts in to syncytiotrophoblasts. The expression of SLPI in BeWo choriocarcinoma cells was inhibited by use of specific oligos designed. Based on the preliminary study two oligos the use of which resulted in maximum inhibition of expression of SLPI more than $75 \%$ by $72 \mathrm{hrs}$ as assessed by RT-PCR and Western blot were employed in this study. Inhibition of SLPI expression by siRNA resulted in inhibition of morphological differentiation of BeWo cells. This was also reflected functionally by increase in the protease activity as assessed by gelatin zymography. The observation that the expression of two differentiation markers namely Endoglin and hCG also decreased following silencing suggest a role for SLPI in differentiation of cytotrophoblasts to syncytiotrophoblast. RT-PCR analysis for the proliferation markers Cyclin A2 and PCNA in the SLPI silenced cells revealed an increase in their expression. In contrast analysis for differentiation markers GADD45A, DNA-PK,ADRP, and MAP Kinase revealed a decrease in their expression. These results suggest an important role for SLPI during differentiation of cytotrophoblasts into syncytiotrophoblasts and are of significance in that silencing of a single gene can disrupt this differentiation process and establish the importance of SLPI during differentiation process per se.
\end{abstract}

Keywords: SLPI, siRNA, Cell Differentiation, Cytotrophoblast, Syncytiotrophoblast

\section{Introduction}

Cellular differentiation is a complex but fascinating process in all multi-cellular organisms. Differentiation can involve changes in numerous aspects of cell physiology; size, shape, polarity, metabolic activity, responsiveness to signals, and changes in gene expression profiles. These changes form the basis for differentiation to occur.

Placental development is directed towards the establishment of a continuous nutrient supply to the developing fetus. This requires efficient access of maternal blood to a transporting surface, the multinucleate syncytiotrophoblast layer. This is made possible by the rapid proliferation and ensuing invasion of mononuclear trophoblasts into the uterus and remodeling of the spiral arteries therein. It is interesting to note that in early pregnancy, it is the placenta that first engages its active growth and proliferation and only then, permits the logarithmic growth phase of the embryo. As a developing organ, the placenta undergoes constant tissue remodeling, which is characterized by the functional loss of trophoblast cells by apoptosis.

BeWo, a cell line derived from human gestational choriocarcinoma, is an extensively used model to understand the molecular changes during the differentiation of cytotrophoblasts (CT) to syncytiotrophoblast (ST) in the placenta [1-6]. The main cell types present in the placenta are CT and ST. CT is highly proliferative and invasive and relatively less functional while ST is non proliferative and non invasive and highly functional. ST is the site of production of a variety of proteins, peptides, steroid hormones and growth factors. Multi-nucleated ST is formed by the fusion of uninucleated CT and this transformation can be induced in vitro by a variety of effectors which include Forskolin, 
TGF $\beta 1$, and other growth factors [7-10].

In our efforts to profile the transcripts during differentiation of CT into ST, we have carried out Differential-Display Reverse Transcription-PCR (DD-RT-PCR) and Microarray analysis of RNA isolated from both cell types [11]. In addition, to the expected change in expression of several genes, the expression of a transcript corresponding to SLPI (secretory leukocyte protease inhibitor) was up regulated several folds in the ST.

SLPI is an $11.7 \mathrm{kDa}$ cationic protein and is a member of the innate immunity-associated proteins. It is a non-glycosylated, highly basic, acid-stable, cysteine-rich, 107-amino acid, single-chain polypeptide [11]. The SLPI gene, along with the elafin gene, is a member of the trappin gene family. The products of this family may cross-link to various proteins by a transglutaminase activity. SLPI has 16 cysteine residues, which form eight disulphide bridges [12] while elafin and trappin-2 have four disulphide bridges. A structure determination by $\mathrm{x}$-ray crystallography showed a boomerang shape, composed of two similar domains each having 54 and 53 amino acids[13], and the active inhibitory site for elastase is in the C-terminal domain[14]. These two domains ( $\mathrm{COOH}$ terminal and $\mathrm{NH} 2$ terminal) share about $35 \%$ identity and are coded by different exons. SLPI performs its anti-protease activity via its $\mathrm{COOH}$-terminal domain, and the active center of which is formed by the Leu72-Met73 residues. The NH2-terminal domain has no similar properties, however it may help in stabilizing the protease-antiprotease complex and may engage the improvement of the anti-proteinase activity of SLPI by heparin. SLPI is a multifunctional protein found in a variety of tissues including placenta, endometrium [15], leucocytes [16], epididymis [17] etc., specifically secreted by the epithelial cells [18] of these tissues and exhibits antimicrobial $[17,19,20]$ and protease inhibitor activity [11]. Considering the several fold up-regulation of SLPI in syncytiotrophoblasts and several functions attributed to SLPI, in the present study we have investigated the role of SLPI during the process of differentiation by silencing of SLPI gene by use of small interfering RNA (siRNA) as an approach.

\section{Materials and Methods}

\subsection{Cell Culture and Induction of Differentiation in $\mathrm{Hu}$ - man Trophoblastic BeWo Cells}

BeWo cells (a human choriocarcinoma cell line, generously gifted by Dr. Susan Fisher, University of California, San Francisco, California) were cultured in DMEM/Ham's F-12 medium (Sigma Chemical Co., St. Louis, MO) containing 10\% FCS (Hyclone laboratories Inc., UT) and antibiotics $(100 \mathrm{U} / \mathrm{ml}$ Penicillin, $50 \mu \mathrm{g} / \mathrm{ml}$ Gentamycin and 5 $\mathrm{U} / \mathrm{ml}$ Nystatin), at $37^{\circ} \mathrm{C}$, in an atmosphere of $5 \% \mathrm{CO} 2$. For experiments involving induction of differentiation, cells were maintained in serum-free medium [as described by Taylor et. al. (1991), with minor modifications] prepared with DMEM/Ham's F-12 medium supplemented with insu- lin $(1 \mu \mathrm{g} / \mathrm{ml})$, transferrin $(5 \mu \mathrm{g} / \mathrm{ml})$, Bovine Serum Albumin $(500 \mu \mathrm{g} / \mathrm{ml})$ and antibiotics.

\section{2. siRNA Design and Synthesis}

Four siRNA duplexes for human SLPI gene (NM_003064) were obtained from Qiagen (HP Guaranteed siRNA). The siRNA duplexes were designed by using Hi-performance Design Algorithm licensed from Novartis $\mathrm{AG}$, integrated with a stringent in-house homology analysis tool. The highest-ranking siRNA duplexes generated by the algorithm were chosen, representing the best combination of activity and specificity. The following sequences of siRNAs were used in this study, SLPI-I [sense-r(CCAGUUGAUCAAUGAAUAA)dTdT; anti-sense r(UUAUUCAUUGAUCAACUGG)dCdA],SLPI-II [sense- r(GCCAAUG-UUUGAUGCUUAA) dTdT; anti-sense-r(UUAAGCAUCAAACAUUGGC)dCdA],SLPI-II I [sense-r(GCUGGAGU CUGUCCUCCUA)dTdT; anti-sense r(UUAAGCAUCAAA-CAUUGGC)dC dT], SLPI-IV [sense-r(GCUGGAGUCUGUCCUCCUA)dTdT; anti-sense r(UUAAGCAUCAAACAUUGGC) dC $\mathrm{dT}$ ]. Individual siRNAs and mixture of all four siRNAs were reconstituted for transfection according to the manufacturer's instructions.

\subsection{Transfection of siRNA}

$3 \times 105$ BeWo cells were plated and grown over night at $37^{\circ} \mathrm{C}$ to $50 \%$ confluence in 6-well plates and were transfected with different oligos (SLPI I, II, III, and IV) using different concentrations ranging from 5-25nM and harvested after the $72 \mathrm{hrs}$ incubation. The extent of silencing of SLPI expression was assessed by RT-PCR analysis. Initially we used each of the four oligos designed at a maximum concentration of $25 \mathrm{nM}$. Control experiments were carried out transfecting the cells with non-silencing siRNA with no homology to any known gene in the human genome. We observed from our transfection studies that SLPI II and SLPI III at $25 \mathrm{nM}$ were capable of inhibiting the expression of SLPI to the extent of $70-90 \%$ individually while with oligo I \& IV only about $50 \%$ inhibition was seen even at $25 \mathrm{nM}$. Since we noticed transfection of a combination of SLPI II and SLPI III was more effective ( $>90 \%$ ), we have decided to employ only these two oligos at $25 \mathrm{nM}$ concentration in our studies to assess the effect of silencing on the syncytium formation and thus differentiation process.

\subsection{RNA Isolation and Semi-Quantitative RT-PCR}

Total RNA was isolated using TRI reagent (Sigma Chemicals Co., MO USA) according to manufacturer's instructions. RNA quality was assessed by formaldehyde-agarose gel electrophoresis. Two microgram of DNAse-I (Amersham Biosciences, U.K) treated RNA was reverse transcribed at $37^{\circ} \mathrm{C}$ with 200 units of MMLV-reverse transcriptase (Amersham Biosciences, U.K.) and random hexamers (Roche Molecular Biochemicals, Germany). An aliquot of $2 \mu \mathrm{l}$ of the cDNA was employed 
for PCR amplification using specific primers. An aliquot of $12 \mu \mathrm{l}$ of the PCR product was electrophoresed on $1.5 \%$ agarose gel containing $0.5 \mu \mathrm{g} / \mathrm{ml}$ ethidium bromide, in a buffer containing $45 \mathrm{mM}$ Tris borate- $1 \mathrm{mM}$ EDTA. The difference in intensities of the products following electrophoresis was analyzed by using EDAS 120 Kodak Gel documentation system. The authenticity of the products was confirmed by sequencing. The expression levels of various genes analyzed were inferred upon normalizing the signal intensities with that of $\beta$-actin, which served as an internal control in this semi-quantitative analysis. Values so obtained were used to determine mean \pm SE for a graphical representation. Primers used for RT-PCR analyses were designed using Primer express software and were obtained from Microsynth GmbH, Switzerland. Details of the primers and the PCR conditions are given in Table 1.

Table. 1. List of primers used.

\begin{tabular}{|c|c|c|c|}
\hline Products & Primers (5'-3') & $\begin{array}{r}\text { Size } \\
\text { (in bp) }\end{array}$ & $\begin{array}{l}\text { Annealing } \\
\text { Temp } \\
\left({ }^{\circ} \mathrm{C}\right)\end{array}$ \\
\hline$\beta$-hCG & $\begin{array}{l}\text { F- gttccaggggetgctgctgtt } \\
\text { R- cgggagtcgggatgggcttgg }\end{array}$ & 457 & 64 \\
\hline PCNA & $\begin{array}{l}\text { F- gctccagcggtgtaaacctgca } \\
\text { R- cgtgcaaattcaccagaaggca }\end{array}$ & 346 & 60 \\
\hline Endoglin & $\begin{array}{l}\text { F-tgtctcacttcatgectc cag ct } \\
\text { R-aggctgtccatgttgaggcagt }\end{array}$ & 378 & 54 \\
\hline Cyclin A2 & $\begin{array}{l}\text { F- cgtggactggttagttga } \\
\text { R- atggcaaatacttgaggt }\end{array}$ & 416 & 54 \\
\hline FASL & $\begin{array}{l}\text { R- ttcttccetgtccaacctctg } \\
\text { R- caatcctaccaaggcaaccag }\end{array}$ & 211 & 57 \\
\hline GADD45A & $\begin{array}{l}\text { F-gcgagaacgacatcaacatcc } \\
\text { R- cgactttcccggcaaaaac }\end{array}$ & 204 & 58 \\
\hline SLPI & $\begin{array}{l}\text { F-gacaccccaaacccaacaa } \\
\text { R-ccccaaaggaggatatcagtg }\end{array}$ & 269 & 60 \\
\hline $\mathrm{Bcl}-2$ & $\begin{array}{l}\text { F-tggcettctttgagttcggt } \\
\text { R-agtgaacgetttgtccagag }\end{array}$ & 312 & 58 \\
\hline Bax & $\begin{array}{l}\text { F-tttcatccaggatcgagca } \\
\text { R-tgccactcggaaaaagacct }\end{array}$ & 208 & 58 \\
\hline DNA-PK & $\begin{array}{l}\text { F-aataccttggaccggttgct } \\
\text { R-agtacttcggaaacgccaatc }\end{array}$ & 205 & 58 \\
\hline MEKK-1 & $\begin{array}{l}\text { F-gatgacagctttggcttgagc } \\
\text { R-tgagacgctgacattgccat }\end{array}$ & 323 & 58 \\
\hline$\beta$-actin & $\begin{array}{l}\text { F- ggcaccacaccttctacaatgagc } \\
\text { R- cgtcatactcctgcttgctgatcca }\end{array}$ & 737 & 60 \\
\hline ADRP & $\begin{array}{l}\text { F-ctgtctaccaagctctgctc } \\
\text { R- cgatgcttctcttccactcc }\end{array}$ & 298 & 57 \\
\hline
\end{tabular}

\subsection{MTT Assay of siRNA Transfected Forskolin Treated BeWo Cells}

Cells $(3 \times 105)$ were plated and grown over night at $37^{\circ} \mathrm{C}$ to $50 \%$ confluence in 6-well culture plates and transfection was carried out according to the manufacturer's instructions using Hi-perfect Transfection reagent kit from Qiagen GmbH Str. 1 Hilden, Germany. Transfection was carried out either with control siRNA or siRNA oligos. $10 \mu \mathrm{M}$ forskolin was added to BeWo to induce differentiation and then incubated for $72 \mathrm{hrs}$. At the end of the incubation period, $10 \mu \mathrm{l}$ of $5 \mathrm{mg} / \mathrm{ml}$ MTT was added to the cultures and further incubated for $3-4 \mathrm{hrs}$ at $37^{\circ} \mathrm{C}$. Following this, the medium was removed and the reaction terminated, by adding $500 \mu 1$ of acidified isopropanol (40mM HCL in absolute isopropanol) to extract the formazon crystals formed. Absorbance of the converted dye was measured at 570nm using a UV Max reader (Molecular devices, Menlo Park, CA, USA). All the tests were performed in triplicates. Data are expressed graphically (Absorbance at $570 \mathrm{~nm}$ as a function of time).

\subsection{Western Blot Analysis}

An equal quantity of protein $(40 \mu \mathrm{g})$ was electrophoresed on $10 \%$ SDS-polyacrylamide gel and transferred onto the nitrocellulose (Sartorius AG, Germany) membrane, using a semi-dry transfer apparatus. After completion of transfer, the membranes were blocked in 5\% non-fat milk in Tris-Buffered-Saline containing $0.05 \%$ Tween-20 (TBST) and incubated overnight at $4^{\circ} \mathrm{C}$ with SLPI antibody $(1: 200$ dilution). The SLPI specific antibody was obtained as described [21]. Membrane was washed several times with TBST and incubated with the appropriate Horse-Radish-Peroxidase (HRP)-conjugated secondary antibody for $1 \mathrm{~h}$. Following extensive washing in TBST, bound antibody was detected by an Enhanced Chemi-Luminiscence (ECL) detection kit (Amersham-Pharmacia Biotech. UK). Following hybridization, the blot was stripped and re-probed (using the protocol recommended by the manufacturer) for $\beta$-actin (Polyclonal antibody from Cell Signaling Technology Inc, Danvers, MA) which served as an internal control for assessing equality of protein loading.

\subsection{Gelatin Zymography}

SDS-PAGE gels $(10 \%)$ were prepared according to the standard procedure $[21,22]$ along with $0.1 \%$ gelatin in the running gel. The sample to be used for checking the presence of protease was mixed with one part of Tris-Glycine SDS Sample Buffer (2X) and was allowed to stand for 10 minutes at room temperature taking care not to heat the samples before subjecting to electrophoresis. After running at 100 volts at $40^{\circ} \mathrm{C}$, the gels were soaked in $10 \%$ Triton $\mathrm{X}$ 100 (renaturing buffer) for 1 hour with gentle agitation. The Zymogram Renaturing Buffer was decanted and replaced with 1x Zymogram Developing buffer [Tris base $(50 \mathrm{mM})$, $\mathrm{NaCl}(0.2 \mathrm{M}), \mathrm{CaCl} 2(5 \mathrm{mM})]$, over night at $37^{\circ} \mathrm{C}$ and Stained with Coomassie Blue R-250 for 30 minutes. In this procedure, areas of protease activity appear as clear bands against a dark blue background where the substrate is digested by the protease present in the extract being analyzed.

\subsection{Statistical Analysis}

All variables were compared using parametric analysis of variance (ANOVA) following verification of distribution normality and homoscedasticity. When data did not fulfill 
these requirements, non parametric Kruskal-Wallis tests were used. Significant ANOVA were followed by a post hoc multiple comparison test (Newman-Keuls), and Kruskal-Wallis test by a rank-based multiple comparisons. All statistical analyses were conducted using Graphpad software (San Deigo, CA, USA), and for all tests, the significant threshold was $\mathrm{P}<0.05$.

\section{Results}

\subsection{Silencing of SLPI Expression by siRNA Inhibits Morphological and Functional Differentiation in BeWo Cells}

BeWo cells were transfected with control siRNA or SLPI II and SLPI III in combination at $25 \mathrm{nM}$ concentration. After $12 \mathrm{~h}$ of incubation at which time point $50 \%$ inhibition was seen with oligo II and III Forskolin $(10 \mu \mathrm{M})$ was added and incubated for $72 \mathrm{~h}$ to induce differentiation.

As can be seen from the results presented in Fig.1A, morphological differentiation was inhibited in cells transfected with SLPI siRNA when compared with control siRNA transfected cells. The formation of syncytium was not seen in SLPI silenced cells. Though the cells were coming closer, the fusion of cells could not be seen following silencing of SLPI expression in the cells.

Results of RT-PCR analysis presented in Fig 1.B confirmed that SLPI expression is indeed inhibited in SLPI siRNA treated cells when compared to control non-silencing oligo transfected cells. It should be recalled that we have demonstrated that during differentiation there is 15 fold increase in SLPI mRNA expression in syncytiotrophoblasts. This up-regulation was not seen in SLPI silenced siRNA treated cells but was seen in control siRNA transfected cells (Fig.1B). Western blot analysis showed that the silencing was effective not only at the mRNA level but also at the SLPI protein level (Fig 1C).

\section{Non-silencing oligo}

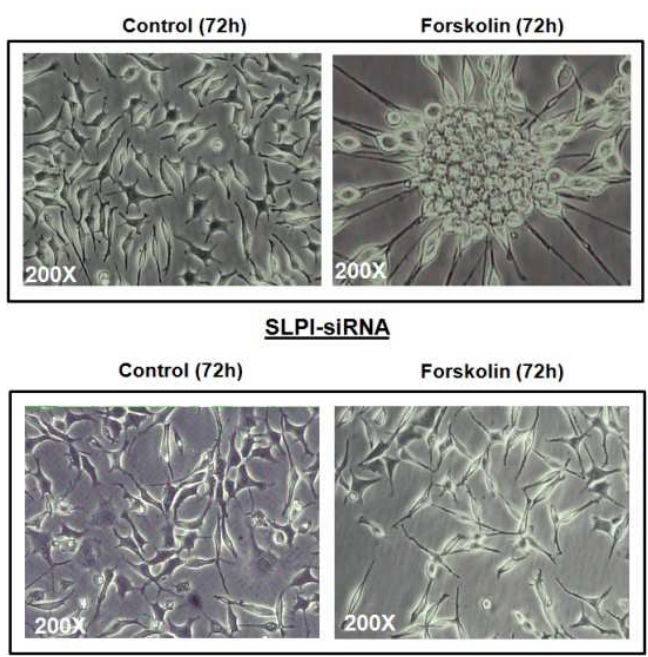

(A)

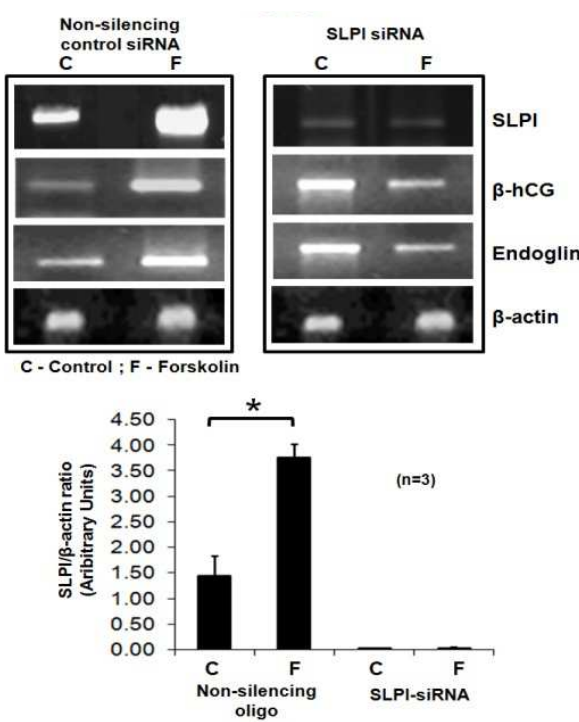

(B)
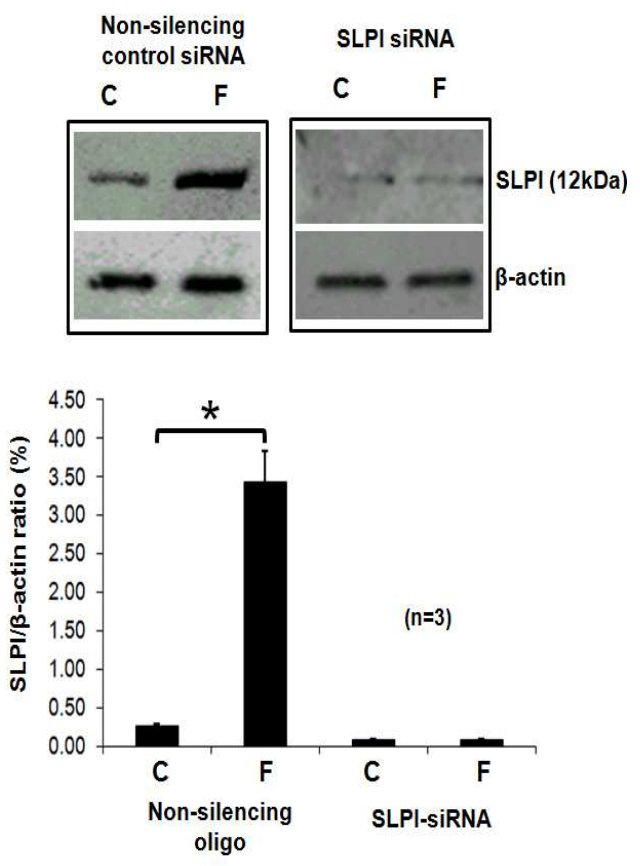

(C)

Figure 1. (A) Morphological changes seen in BeWo cells transfected with non-silencing and silencing siRNA (SLPI II \& III at 25nM) incubated with Forskolin for $72 h$. (B) RT-PCR analysis for SLPI, differentiation markers such as $\beta$ - $h C G$ and Endoglin. RNA was isolated from control and SLPI silencing siRNA transfected cells, cDNA subjected to semi-quantitative $P C R$ in the linear range of amplification with $\beta$-actin as an internal control. Graphical representation of data used as an internal control (values represent relative levels, mean $\pm S$.E. from three independent experiments). (C) Western blot analysis for SLPI after SLPI silencing and Forskolin treatment. Western blot analysis of SLPI in proteins from control siRNA and SLPI (SLPI II \& III at 25nM) silenced cells treated with forskolin for 72h. Antiserum to SLPI was used at a dilution of 1:200 and Ag-Ab complex was detected using ECL Kit (Amersham Biosciences Ltd., Hong Kong). Panel C provides graphical representation of data. $\beta$-actin was used as an internal control. Values shown are mean \pm S.E from three independent experiments $(* p<0.05)$. 
In order to ascertain whether this inhibition of morphological differentiation also resulted in the inhibition of functional differentiation we checked for the expression of $\beta$-hCG and Endoglin which are well-established differentiation markers for the differentiated BeWo cells. The results presented in Fig.1B clearly establish that there is an increase in $\beta$-hCG and Endoglin expression in control (non-silencing siRNA oligo transfected) Forskolin treated cells but not in SLPI silenced (siRNA treated) Forskolin treated cells. The viability of the cells was assessed by MTT assay at end of the each incubation which was unaffected as can be seen from the results in Fig.2.

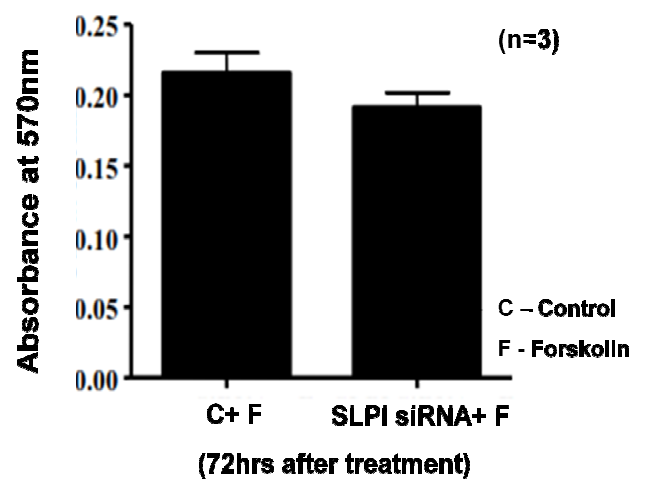

Figure 2. MTT assay of siRNA transfected Forskolin treated BeWo cells. Cells $\left(3 \times 10^{5}\right)$ were plated and grown over night at $37^{\circ} \mathrm{C}$ to $50 \%$ confluence in 6 well plates and transfection was carried out. For the induction of differentiation in SLPI silenced BeWo cells, transfection was carried out with $25 \mathrm{nM}$ of SLPI-II and III SiRNA and $12 \mathrm{~h}$ after transfection $10 \mu \mathrm{M}$ of Forskolin was added and incubated the cells for $72 \mathrm{~h} . \quad$ MTT assay was carried out for cells transfected with non-silencing oligo or SLPI SiRNA and later incubated with Forskolin for 72 h. Values shown are mean $\pm S . E$ from three independent experiments.

\subsection{Silencing of SLPI Expression Increases Protease Ac- tivity in BeWo Cells}

Since SLPI is a protease inhibitor, and it is known that proteases and their inhibitors play an important role during differentiation, it was of interest to verify whether silencing of the expression SLPI mRNA is also reflected at the functional level also. This was assessed by analyzing the protein extracts made from control and SLPI silenced cells after treatment with forskolin by Gelatin Zymography. It can be seen from the results presented in Fig. 3 that there is a small increase in protease activity in SLPI silenced cells compared to control cells in which SLPI expression is intact. The increase in protease activity is assessed by the intensity of the white bands that is due to the proteases which digest the gelatin in the gel when incubated with appropriate buffer at $37^{\circ} \mathrm{C}$. It is to be noted that no effort in the present study has been made to establish the identity of the enzymes (possibly Elastase and Cathepsin G) whose activity is increased following silencing of SLPI. However it is well established that SLPI is a protease inhibitor as such increase in protease activity as assessed by increase in intensity of the white bands supports the conclusion that there is increase in the protease activity following silencing of expression of SLPI.

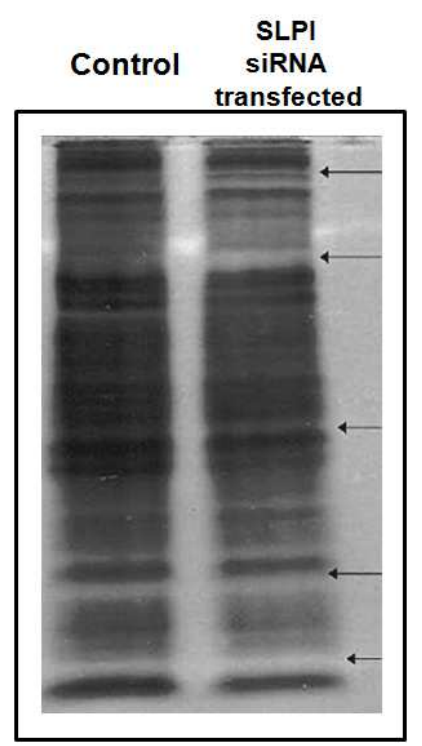

Figure. 3. Gelatin Zymography for control and SLPI siRNA transfected and Forskolin treated cells. Protein samples obtained from control siRNA and SLPI siRNA transfected and Forskolin treated cells were subjected to $10 \%$ SDS PAGE containing $0.1 \%$ gelatin. Gelatin Zymography was carried out as described in materials and methods. The arrows indicate the increase in the protease activity.

\subsection{Genes Involved in Cell Cycle, Proliferation, Differen- tiation and Apoptosis}

Our previous study involving DD-RT-PCR and microarray analysis of forskolin induced differentiation in BeWo cells have been able to identify several genes which have been grouped in to four categories based on their functional importance like cell proliferation/differentiation, apoptosis, cell migration, invasion and adhesion, inflammatory pathway and we were also able to relate these genes to the functions involved during implantation, placentation and differentiation of trophoblastic cells. Considering this, it was of interest to verify these observations employing the model system we have developed, where in silencing of a single gene results in arrest of differentiation. As can be seen from the results of the RT-PCR analysis presented in Fig.4, GADD45A (Growth arrest and DNA-damage-inducible protein alpha), ADRP (Adipose differentiation-related protein) were down regulated when SLPI was silenced. It should be noted that these genes are differentiation markers of trophoblasts and expression profile of these genes is exactly reverse of the profile seen when SLPI was not silenced. DNA-protein kinase and MAP kinase (p38) genes were also down regulated when SLPI gene was silenced and differentiation was inhibited when compared to the other model system where SLPI was intact. Proliferation arrest is one of pre-requisites before differentiation starts and as can be seen from the results presented in Fig.4 both Cyclin-A2 and PCNA (Proliferating Cell Nuclear Antigen) which are well documented proliferation markers are up-regulated when SLPI is silenced which clearly establishes that proliferation 
is not arrested.

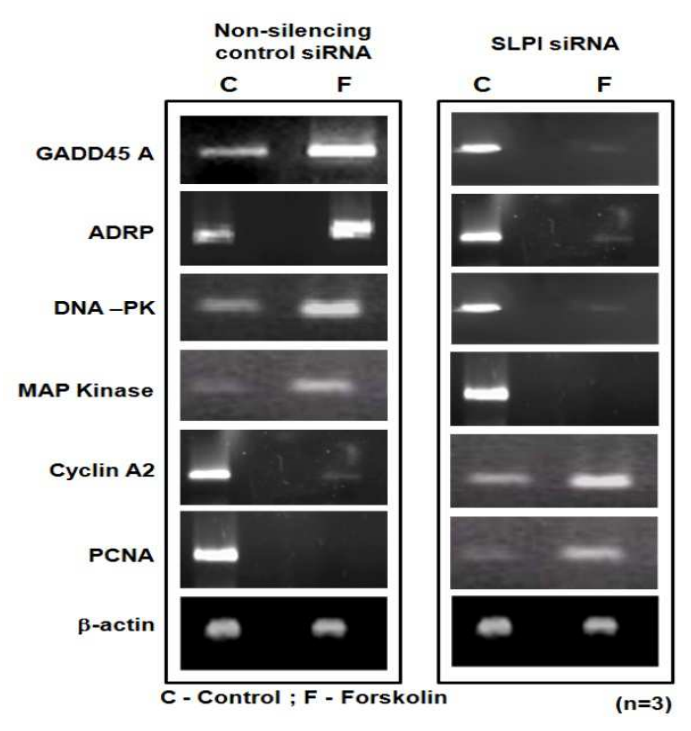

(A)

(B)

Figure.4. RT-PCR analysis for genes related to cell cycle, proliferation and differentiation genes. Total RNA isolated from control and non-silencing siRNA Forskolin treated cells (A), Control siRNA and SLPI siRNA transfected and Forskolin treated cells (B) analyzed for the expression GADD45A, ADRP, MAP Kinase, CyclinA2 and PCNA in the linear range of amplification with $\beta$-actin as an internal control by semi-quantitative RTPCR.

As can be seen from Fig.5, RT-PCR analysis of bcl-2 (B-cell lymphoma 2) was down-regulated and Bax was up-regulated in SLPI intact Forskolin induced differentiated cells. But in SLPI silenced Forskolin treated cells, Bcl-2 was up regulated and BAX (Bcl-2-associated X protein) was down regulated. It is known that up- regulation of apoptotic genes takes place when cells differentiate in to syncytiotrophoblasts.

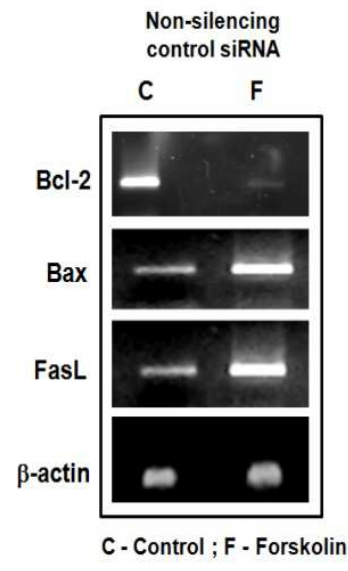

(A)

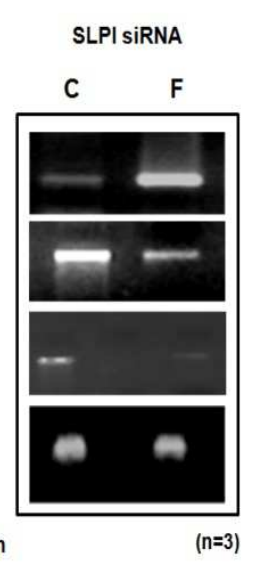

(B)
Figure. 5. RT-PCR analysis for genes involved in apoptosis pathway. Total RNA isolated from control and non-silencing siRNA Forskolin treated cells (A), Control siRNA and SLPI siRNA transfected and Forskolin treated cells (B) analyzed for the expression Bcl2, Bax and FasL with $\beta$-actin as an internal control by semi-quantitative RTPCR.

\section{Discussion}

In the present study we have employed BeWo cell line for blocking the expression of SLPI by siRNA. Although the use of cells isolated from human placenta to silence expression of SLPI is more ideal, most of the published studies using siRNA to silence the expression of genes in human placental cells have been carried out using cell lines. It has to be noted that the process of maintaining primary cells isolated from fresh human placenta transfection studies is time consuming and beset with problems of yeast contamination during the course of culture.

SLPI is a multifunctional protein found in a variety of tissues including placenta, endometrium [15], leucocytes [16], epididymis [17], etc., specifically secreted by the epithelial cells [18] of these tissues and exhibits antimicrobial $[17,19,20]$ and protease inhibitor activity [11]. It is likely that the anti-protease and anti-microbial action of this molecule is involved in key reproductive events such as fertilization, implantation and pregnancy. Considering our observation that the expression of SLPI mRNA was up regulated several fold [21] in syntiotrophoblasts it was of interest to evaluate the effect of blocking the expression of SLPI by silencing the expression of SLPI gene.

Following a preliminary study, four siRNA oligos were designed to target different regions of SLPI mRNA and it was found that all the siRNAs individually or in combination were effective in silencing the expression of SLPI by $>80 \%$ at mRNA level as assessed by RT-PCR analysis. The effect of silencing is observed even after $72 \mathrm{~h}$ of transfection and control experiment was carried out by transfecting the cells with non-silencing siRNA with no homology to any known gene in the human genome. We employed oligo II and III which were highly efficient in silencing the SLPI mRNA expression for our differentiation experiments. The cells were initially transfected with oligo-II and III after $12 \mathrm{~h}$, Forskolin was added to induce differentiation.

The results revealed that transfection with oligo-II and III siRNA resulted in a significant decrease in the expression of SLPI both at mRNA and protein level compared to the control cells in which non silencing siRNA was transfected prior to the addition of Forskolin. While multinucleated syncytiotrophoblasts were seen in control cells, it was not observed in SLPI silenced cells (Fig.1A). Inhibition of differentiation was also confirmed by the fact that there was a marked decrease in the level of $\beta$-hCG and endoglin which are functional markers of differentiation expressed in syncytiotrophoblasts.

As mentioned in the results section inhibition of differentiation was established not just by change in the morphology and decrease in the level of mRNA and protein but also by a functional parameter. A significant increase in protease activity in SLPI silenced cells compared to control cells in which SLPI expression is intact was seen. RT-PCR analysis of RNA from control vector transfected and cells in which SLPI expression was silenced revealed that the level of expression of all the genes associated with proliferation 
such Cyclins, PCNA etc were up regulated in the group in which expression was silenced. Although the western blot data for the above markers like Cyclin, PCNA etc., would have provided additional evidence for the role of SLPI in the differentiation process, the results obtained using RTPCR analysis we feel are quite convincing. The possibility that the inhibition of expression of SLPI following transfection of siRNA is not due to toxicity was established by the MTT assay, before and after transfection which revealed that viability was unaffected.

Proteases and protease inhibitors play a very crucial role in tissue and membrane remodeling and wound healing and in invasive processes [23-25]. During placentation, villi invade into the maternal endometrium and during this period there is considerable tissue remodeling and degradation. Several types of proteases are involved in this invasion process and action of these proteases should be controlled and regulated [26, 27]. It has been suggested that the extent of embryo - maternal interaction and hence the nature of placentation is dependent upon the level and type of proteases and protease inhibitors [27,28]. SLPI, a protease inhibitor present in the syncytiotrophoblast may have a crucial role in controlling this process $[15,29,30]$. It has been suggested that SLPI acts to maintain the uterine-placental border. It is also present in uterine fluid [31]. Successful human pregnancy demands that inflammatory response [32] in the uterus is suppressed and SLPI as an anti bacterial and anti inflammatory molecule may contribute to this. Differentiation is a complex process which involves changes in several aspects of cell physiology; size, shape, polarity, metabolic activity, responsiveness to signals, and changes in gene expression profiles etc. It is interesting to note that silencing of expression of a single gene SLPI results in the inhibition of such a complex process of differentiation.

\section{Conclusion}

It was observed that in BeWo choriocarcinoma cells the silencing of expression of SLPI mRNA a transcript whose expression was up-regulated several folds in syncytiotrophoblast resulted in inhibition of differentiation of cytotrophoblast in to syncytiotrophoblast. The fact that the expected morphological changes following induction of differentiation by addition of forskolin to the cytotrophoblast were not seen also supports role for SLPI in the differentiation. In addition the observation that the expected changes in the expression of proliferation and differentiation markers as assessed by RT-PCR were also not seen supports role for SLPI in differentiation of CT to ST. Also the silencing of expression of SLPI resulted in an increase in protease activity as assessed by gelatin zymography.

\section{Acknowledgment}

The authors wish to acknowledge the help received from Dr. (Mrs.) S. Rama and Dr. M. Anbalagan during the initial phases of the work. One of the authors, A.J. Rao is thankful to the Department of Science and Technology for Rajaramanna fellowship. The authors also wish to thank Indian Institute of Science, Bangalore, Indian Council of Medical Research (Govt. of India), Mellon Foundation, USA and CONRAD, USA for the financial assistance provided during the course of the work.

\section{References}

[1] Lyden TW, Ng AK, Rote NS. Modulation of phosphatidylserine epitope expression by BeWo cells during forskolin treatment. Placenta. 14 (1993) 177-186.

[2] Vogt E, Ng AK, Rote NS. Antiphosphatidylserine antibody removes annexin- $\mathrm{V}$ and facilitates the binding of prothrombin at the surface of a choriocarcinoma model of trophoblast differentiation. Am J Obstet Gynecol. 177 (1997) 964-972.

[3] Rote NS, Chang J, Katsuragawa H, Ng AK, Lyden TW, et al. Expression of phosphatidylserine-dependent antigens on the surface of differentiating BeWo human choriocarcinoma cells. Am J Reprod Immunol. 33 (1995) 114-121.

[4] Rote NS, Vogt E, DeVere G, Obringer AR, Ng AK. The role of placental trophoblast in the pathophysiology of the antiphospholipid antibody syndrome. Am J Reprod Immunol. 39 (1998) 125-136.

[5] Lin L, Xu B, Rote NS. Expression of endogenous retrovirus ERV-3 induces differentiation in BeWo, a choriocarcinoma model of human placental trophoblast. Placenta. 20 (1999) 109-118.

[6] $\mathrm{Xu} \mathrm{B}$, Lin L, Rote NS. Identification of a stress-induced protein during human trophoblast differentiation by differential display analysis. Biol Reprod. 61 (1999) 681-686.

[7] Garcia-Lloret MI, Morrish DW, Wegmann TG, Honore L, Turner AR, et al. Demonstration of functional cytokine-placental interactions: CSF-1 and GM-CSF stimulate human cytotrophoblast differentiation and peptide hormone secretion. Exp Cell Res. 214 (1994) 46-54.

[8] Yang M, Lei ZM, Rao Ch V. The central role of human chorionic gonadotropin in the formation of human placental syncytium. Endocrinology. 144 (2003) 1108-1120.

[9] Rama S, Petrusz P, Rao AJ. Hormonal regulation of human trophoblast differentiation: a possible role for 17beta-estradiol and GnRH. Mol Cell Endocrinol. 218 (2004) 79-94.

[10] Rama S, Suresh Y, Rao AJ. TGF betal induces multiple independent signals to regulate human trophoblastic differentiation: mechanistic insights. Mol Cell Endocrinol. 206 (2003) 123-136.

[11] Neelima PS, Rao AJ. Gene expression profiling during Forskolin induced differentiation of BeWo cells by differential display RT-PCR. Mol Cell Endocrinol. 281 (2008) 37-46.

[12] Thompson RC, Ohlsson K. Isolation, properties, and complete amino acid sequence of human secretory leukocyte protease inhibitor, a potent inhibitor of leukocyte elastase. Proc Natl Acad Sci. 83 (1986) 6692-6696.

[13] Schalkwijk J, Wiedow O, Hirose S. The trappin gene family: proteins defined by an $\mathrm{N}$-terminal transglutaminase substrate 
domain and a C-terminal four-disulphide core. Biochem J. 340 (1999) Pt 3.: 569-577.

[14] Grutter MG, Fendrich G, Huber R, Bode W. The 2.5 A X-ray crystal structure of the acid-stable proteinase inhibitor from human mucous secretions analysed in its complex with bovine alpha-chymotrypsin. Embo J. 7 (1988) 345-351.

[15] Eisenberg SP, Hale KK, Heimdal P, Thompson RC. Location of the protease-inhibitory region of secretory leukocyte protease inhibitor. J Biol Chem. 265 (1990) 7976-7981.

[16] King AE, Critchley HO, Kelly RW. Presence of secretory leukocyte protease inhibitor in human endometrium and first trimester decidua suggests an antibacterial protective role. Mol Hum Reprod. 6 (2000) 191-196.

[17] Bohm B, Aigner T, Kinne R, Burkhardt H. The serine-protease inhibitor of cartilage matrix is not a chondrocytic gene product. Eur J Biochem. 207 (1992) 773-779.

[18] Ohlsson K, Bjartell A, Lilja H. Secretory leucocyte protease inhibitor in the male genital tract: PSA-induced proteolytic processing in human semen and tissue localization. J Androl. 16 (1995) 64-74.

[19] Xu W, He B, Chiu A, Chadburn A, Shan M, et al. Epithelial cells trigger frontline immunoglobulin class switching through a pathway regulated by the inhibitor SLPI. Nat Immunol. 8 (2007) 294-303.

[20] Tomee JF, Hiemstra PS, Heinzel-Wieland R, Kauffman HF. Antileukoprotease: an endogenous protein in the innate mucosal defense against fungi. J Infect Dis. 176 (1997) 740-747.

[21] McNeely TB, Dealy M, Dripps DJ, Orenstein JM, Eisenberg SP, et al. Secretory leukocyte protease inhibitor: a human saliva protein exhibiting anti-human immunodeficiency virus 1 activity in vitro. J Clin Invest. 96 (1995) 456-464.

[22] Nampoothiri LP, Neelima PS, Rao AJ. Proteomic profiling of forskolin-induced differentiated BeWo cells: an in-vitro model of cytotrophoblast differentiation. Reprod Biomed
Online. 14 (2007) 477-487.

[23] Graham CH, Lala PK. Mechanism of control of trophoblast invasion in situ. J Cell Physiol. 148 (1991) 228-234.

[24] Page-McCaw A, Ewald AJ, Werb Z. Matrix metalloproteinases and the regulation of tissue remodelling. Nat Rev Mol Cell Biol. 8 (2007) 221-233.

[25] Ashcroft GS, Lei K, Jin W, Longenecker G, Kulkarni AB, et al. Secretory leukocyte protease inhibitor mediates non-redundant functions necessary for normal wound healing. Nat Med. 6 (2000) 1147-1153.

[26] Salamonsen LA. Role of proteases in implantation. Rev Reprod. 4 (1999) 11-22.

[27] Salamonsen LA, Nie G. Proteases at the endometrial-trophoblast interface: their role in implantation. Rev Endocr Metab Disord. 3 (2002) 133-143.

[28] Roberts RM, Bazer FW. The functions of uterine secretions. J Reprod Fertil. 82 (1988) 875-892.

[29] Badinga L, Michel FJ, Simmen RC. Uterine-associated serine protease inhibitors stimulate deoxyribonucleic acid synthesis in porcine endometrial glandular epithelial cells of pregnancy. Biol Reprod. 61 (1999) 380-387.

[30] Shimoya K, Moriyama A, Ogata I, Nobunaga T, Koyama M, et al. Increased concentrations of secretory leukocyte protease inhibitor in peritoneal fluid of women with endometriosis. Mol Hum Reprod. 6 (2000) 829-834.

[31] Helmig R, Uldbjerg N, Ohlsson K. Secretory leukocyte protease inhibitor in the cervical mucus and in the fetal membranes. Eur J Obstet Gynecol Reprod Biol. 59 (1995) 95-101.

[32] Ness RB. The consequences for human reproduction of a robust inflammatory response. Q Rev Biol. 79 (2004) 383-393. 